\title{
How Intensive are Patients' Behavior in Cost-Related Medication Non- Adherence?
}

\section{James X Zhang*}

Department of Medicine, Section of Hospital Medicine, The University of Chicago, Chicago, USA

*Corresponding author: James X Zhang, Department of Medicine, Section of Hospital Medicine, 5841 South Maryland Avenue, MC 5000, Illinois 60637, USA, Tel: 17738341631; E-mail: xzhang1@medicine.bsd.uchicago.edu

Rec date: Jul 26, 2017; Acc date: Jul 28, 2017; Pub date: Aug 02, 2017

Copyright: (c) 2017 Zhang JX. This is an open-access article distributed under the terms of the Creative Commons Attribution License, which permits unrestricted use, distribution, and reproduction in any medium, provided the original author and source are credited.

\section{Editorial}

The cost barrier to medications is a persistent and serious problem among the U.S. elderly population. Twenty-six percent of the elderly do not take medication as prescribed due to a cost barrier [1]. This is not surprising as many elderly patients depend on a small amount of fixed income from social security and struggle on a day-to-day basis to make difficult choices between meeting the basic needs of daily life and filling prescriptions. The Medicare Part D program, a subsidized drug program, aims to significantly improve access to outpatient prescription drug benefits for millions of elderly Americans [2]. While overall utilization increased after the institution of Part D, [3] costrelated medication non-adherence (CRN) has not deceased or has even worsened among the sickest patients, including those with multiple chronic conditions, depression, and stroke survivors [4-6].

There is robust discussion about the causes and consequences of CRN. Several risk factors for CRN have been identified, including outof-pocket payments, gender, race/ethnicity, insurance coverage, comorbidities, and mental health [7-9]. Research has also shown that increased drug costs adversely affects health outcomes, increases resource use in hospital care and emergency department, raises downstream costs to treat, and imposes heavy avoidable health care costs on society [10-12]. Often, poor health leads to high out-of-pocket payments (OOPs) that results in CRN, and CRN leads to worsening health outcomes, followed by even higher OOPs and then more pervasive CRN behaviors.

Thus, far the literature is strong on the overall prevalence rate of CRN (i.e. the percentage of patients who have any kind of CRN behaviors), but is relatively light on the intensity of CRN behaviors (i.e. for those with CRN, how many have multiple of such behaviors, and what combinations of such behaviors they have). Specifically, a patient can have one or more CRN behaviors: delaying filling prescription, not filling the prescriptions, skipping doses, or splitting doses in order to avoid drug costs. Those with persistent, multiple CRN behaviors will certainly have higher risk of worse health outcomes than those with occasional CRN behavior. Without distinguishing those with pervasive, persistent patterns of CRN from those with occasional CRN behaviors, we may risk targeting an overly expansive population where the intervention is less effective and more expensive. While little is known in this regard, one recent study suggests that in a population at high risk of hospitalization, there are differential levels of intensity of CRN behaviors: for example, among patients reporting CRN, 65\% reported two or more CRN behaviors, while $30 \%$ reported three or more CRN behaviors, and 22\% reported all four types of CRN behaviors [13]. This shows how intensive the CRN behavior is for a significant portion of patients when having cost barriers to medications, and it also suggests the opportunity to target the patients who are mostly likely to benefit from the interventions.

Another angle to explore the intensity of CRN behaviors will be to examine the frequency of such behaviors. For example, patients repeatedly delaying filling of prescriptions, splitting doses, or skipping doses may be at higher risk of poor health outcomes. The current oneitem survey instrument or 4-item survey instruments do not allow us to identify such patients $[14,15]$. While medication possession ratio or proportion of days covered may show some aspects of the intensity of CRN, these measures do not have the capacity to distinguish costrelated medication non-adherence from other types of medication non-adherence that are due to side effects, belief, or cultural reasons. More research is much needed to develop screening tools to identify patients with high intensity of CRN behaviors.

Research shows that successful interventions to address medication non-adherence are generally substantially complex and costly [16]. This calls more research to distinguish various types of non-adherence behaviors, so that more effective, targeted interventions can be developed. These efforts should include the development of screening tools to identify high-risk patients, understand the root causes of CRN behaviors, and design interventions to target patients, reduce CRN behaviors, and improve health outcomes.

\section{References}

1. Wilson IB, Schoen C, Neuman P, Strollo MK, Rogers WH, et al. (2007) Physician-patient communication about prescription medication nonadherence: A 50-state study of America'seniors. J Gen Intern Med 22: 6-12.

2. https://www.cms.gov/medicare/prescription-drug-coverage/ prescriptiondrugcovgenin/

3. Yin W, Basu A, Zhang JX, Rabbani A, Meltzer DO, et al. (2008) The effect of the medicare part $\mathrm{D}$ prescription benefit on drug utilization and expenditures. Ann Intern Med 148: 169-177.

4. Madden JM, Graves AJ, Zhang F, Adams AS, Briesacher BA, et al. (2008) Cost-related medication nonadherence and spending on basic needs following implementation of Medicare Part D. JAMA 299: 1922-1928.

5. Naci H, Soumerai SB, Ross-Degnan D, Zhang F, Briesacher BA (2014) Medication affordability gains following Medicare Part D are eroding among elderly with multiple chronic conditions. Health Aff 33: 1435-1443.

6. Zivin K, Madden JM, Graves AJ, Zhang F, Soumerai SB (2009) Cost-related medication nonadherence among beneficiaries with depression following Medicare Part D. Am J Geriatr Psychiatry 17: 1068-1076.

7. Gellad WF, Haas JS, Safran DG (2007) Race/ethnicity and nonadherence to prescription medications among seniors: Results of a national study. J Gen Intern Med 22: 1572-1578.

8. Piette JD, Heisler M, Wagner TH (2004) Cost-related medication underuse among chronically ill adults: The treatments people forgo, how often, and who is at risk. Am J Public Health 94: 1782-1787. 
Citation: Zhang JX (2017) How Intensive are Patients' Behavior in Cost-Related Medication Non-Adherence?. J Gerontol Geriatr Res 6: e144. doi:10.4172/2167-7182.1000e144

Page 2 of 2

9. Steinman MA, Sands LP, Covinsky KE (2001) Self-restriction of medications due to cost in seniors without prescription coverage. J Gen Intern Med 16:793-799.

10. Goldman DP, Joyce GF, Zheng Y (2007) Prescription drug cost sharing Associations with medication and medical utilization and spending and health. JAMA 298: 61-69.

11. Tamblyn R, Laprise R, Hanley JA, Abrahamowicz M, Scott S, et al. (2001) Adverse events associated with prescription drug cost-sharing among poor and elderly persons. JAMA 285: 421-429.

12. Mojtabai R, Olfson M (2003) Medication costs, adherence, and health outcomes among Medicare beneficiaries. Health Aff 22: 220-229.
13. Zhang JX, Meltzer DO (2016) Identifying patients with cost-related medication non-adherence: A big-data approach. J Med Econ 19: 806-811.

14. http://hrsonline.isr.umich.edu/

15. Soumerai SB, Pierre-Jacques M, Zhang F, Ross-Degnan D, Adams AS, et al. (2006) Cost-related medication nonadherence among elderly and disabled Medicare beneficiaries: A national survey 1 year before the Medicare drug benefit. Arch Intern Med 166: 1829-1835.

16. Osterberg L, Blaschke T (2005) Adherence to medication. N Engl J Med 353: 487-497. 